\title{
Efeito da dexmedetomidina sobre a arritmia cardíaca induzida pela adrenalina em cães anestesiados pelo sevofluorano
}

[Effect of dexmedetomidine on the heart arrhythmia induced by the adrenaline in dogs anesthetized by sevoflurane]

\author{
V.F. Barbosa ${ }^{1}$, N. Nunes ${ }^{2 *}$, E.D.V. Conceição $o^{1}$, C.T. Nishimori ${ }^{1}$, D.P. Paula ${ }^{1}$, \\ P.C. Ferro ${ }^{1}$, R. Carareto ${ }^{1}$. \\ ${ }^{1}$ Aluna de pós-graduação - FCAV-UNESP - Jaboticabal, SP \\ ${ }^{2}$ Faculdade de Ciências Agrárias e Veterinárias - UNESP \\ Via de Acesso Prof. Paulo Donato Castellane, $\mathrm{s} / \mathrm{n}$ \\ 14884-900 - Jaboticabal, SP
}

\begin{abstract}
RESUMO
Avaliou-se o efeito da dexmedetomidina sobre o ritmo cardíaco em 20 cães, sem raça definida, de ambos os sexos e considerados sadios, anestesiados pelo sevofluorano e submetidos a doses crescentes de adrenalina. Os animais foram, aleatoriamente, distribuídos em dois grupos (placebo e dexmedetomidina). No grupo placebo, os animais receberam, por via intravenosa, solução de $\mathrm{NaCl}$ a $0,9 \%$, na dose de $0,3 \mathrm{ml} / \mathrm{kg}$. Foram considerados dois momentos, M0 e M1, imediatamente antes e após a aplicação, respectivamente. Após 10 minutos, realizou-se a indução anestésica com sevofluorano, por meio de máscara facial vedada, até a perda do reflexo laringotraqueal. Em seguida, procedeu-se à intubação orotraqueal e a manutenção da anestesia foi realizada com a administração de sevofluorano na concentração de 1,5CAM, em circuito anestésico com reinalação parcial de gases. Decorridos 20 minutos da indução anestésica, iniciou-se a administração intravenosa contínua de solução de adrenalina a $2 \%$ em doses crescentes de 1, 2, 3, 4 e $5 \mu \mathrm{g} / \mathrm{kg} / \mathrm{min}$, por meio de bomba de infusão, com aumento da dose em intervalos de 10 minutos. Imediatamente antes desse acréscimo eram feitas as mensurações (M2 a M6). No grupo dexmedetomidina empregou-se a mesma metodologia substituindo-se a solução de $\mathrm{NaCl}$ a $0,9 \%$ por hidrocloridrato de dexmedetomidina, na dose de $1 \mu \mathrm{g} / \mathrm{kg}$. Foram registradas as pressões arteriais, em M0 e em M2 a M6, e o traçado eletrocardiográfico, na derivação DII (M2 a M6), considerando-se para efeito estatístico o número total de bloqueios atrioventriculares (BAV) de primeiro e segundo graus e de complexos ventriculares prematuros (ESV), coincidentes com cada dose de adrenalina. Os dados foram submetidos à análise de variância seguida pelo teste de Tukey $(\mathrm{P}<0,05)$. Verificou-se que a dexmedetomidina interfere significativamente na condução atrioventricular levando a maior ocorrência de BAV e reduz o número de ESV nas doses infundidas de 2 e $3 \mu \mathrm{g} / \mathrm{kg} / \mathrm{min}$ de adrenalina. Logo após a aplicação de dexmedetomidina, observaram-se redução da freqüência cardíaca e da pressão arterial, cuja diminuição persistiu por até uma hora.
\end{abstract}

Palavras-chave: cão, alfa-2 agonistas, ritmo cardíaco, anestesia inalatória

\section{ABSTRACT}

The effect of dexmedetomidine on the cardiac rhythm in twenty healthy mongrel dogs of both sexes anesthetized with sevofluorane and submitted to increasing doses of adrenaline was evaluated. The animals were randomly allotted to different treatment groups. Animals of placebo group were intravenous injected with $0.9 \% \mathrm{NaCl}$ solution at $0.3 \mathrm{ml} / \mathrm{kg} / \mathrm{IV}$. Two moments were considered, M0 and $\mathrm{Ml}$, the moments immediately before and after application, respectively. Ten minutes later, the dogs were anesthetized using sevofluorane via face mask until lost of their laringotracheal reflex. Then, orotracheal

Recebido em 12 de fevereiro de 2007

Aceito em 17 de setembro de 2007

*Autor para correspondência (corresponding author)

E-mail: newton@fcav.unesp.br 
intubation was performed and maintenance of anesthesia was kept with 1,5MAC sevofluorane using an anesthetic circuit with a rebreathing system. Twenty minutes after anesthesia induction, continuous IV administration of $2 \%$ adrenaline solution was given at increasing doses of 1,2,3,4 and $5 \mu \mathrm{g} / \mathrm{kg} / \mathrm{min}$., every ten minutes, respectively. The moments M2 to M6 were measured immediately before the next increase of dose. In dexmedetomidine group, the same technique was used replacing $0.9 \% \mathrm{NaCl}$ by dexmedetomidine hydrochloride at $1 \mu \mathrm{g} / \mathrm{kg}$. Blood pressures were recorded at M0 and M2 to M6. Electrocardiography line in the derivation DII (M2 to M6) was used to observe the number of atrioventricular blocks (AVB) of first and second degrees and ventricular premature complexes (VPC). Statistic evaluation was performed by analysis of variance followed by Tukey's test $(P<0.05)$. Dexmedetomidine significantly altered the atrioventricular conduction resulting in a higher occurrence of AVB. This drug reduced the number of $V P C$ at 2 and $3 \mu \mathrm{g} / \mathrm{kg} / \mathrm{min}$ of adrenaline. After administration of dexmedetomidine, reduction of arterial blood pressure up to one hour and reduction of cardiac rate were observed.

Keywords: dog, alpha-2 agonists, cardiac rhythm, inhalatory anesthesia

\section{INTRODUÇ̃̃O}

Os agentes empregados na medicação préanestésica são úteis para preparar o paciente para a anestesia, diminuindo a incidência de efeitos adversos e tornando o ato anestésico o menos desagradável possível, sobretudo para pacientes idosos ou com doenças intercorrentes.

Os agentes agonistas dos receptores alfa-2 adrenérgicos atuam no sistema nervoso central produzindo sedação, relaxamento muscular, ataxia e analgesia. As principais alterações cardiovasculares incluem diminuição da freqüência cardíaca, redução do débito cardíaco, aumento inicial da pressão arterial seguido de hipotensão (Fantoni e Cortopassi, 2002; Tranquilli, 2004) e indução de efeitos arritmogênicos sendo os mais comuns bradicardia, bloqueio sinoatrial e atrioventricular de $1^{\circ}$ e $2^{\circ}$ graus, como resultado da alteração da atividade simpática e parassimpática nas células de condução atrioventricular e da despolarização espontânea dos tecidos do átrio (Tranquilli, 2004).

O cloridrato de dexmedetomidina é um agonista dos receptores alfa-2 adrenérgicos de elevadas seletividade e potência (Scheinin e Virtanen, 1989). Após sua administração em pacientes humanos, a pressão arterial e a freqüência cardíaca apresentaram-se diminuídas, seguidas de sedação. Segundo Sabbe et al. (1994) a dexmedetomidina administrada por via intravenosa, em diferentes doses $(1-10 \mu \mathrm{g} / \mathrm{kg})$ resulta em redução no ritmo cardíaco, sendo que a persistência desse efeito é dependente da dose e pode ser observada por até 2 horas. Kuusela et al. (2001) relataram aumento da sedação e da analgesia com o acréscimo da dose do fármaco.

Os anestésicos inalatórios alteram a função cardiovascular e a magnitude dessa alteração depende, sobretudo, do agente e da dose empregada. Segundo Ebert (1996) e Hettrick et al. (1996), o sevofluorano parece não estar associado ao aumento da freqüência cardíaca quando utilizado em diferentes concentrações em relação a outros agentes anestésicos inalatórios. Gabas et al. (2006) verificaram redução da freqüência cardíaca em cadelas anestesiadas com sevofluorano. Bernard et al. (1990); Lowe et al. (1996) e Mutoh et al. (1997) observaram aumento da freqüência cardíaca basal de 30 a $40 \%$, em cães, durante a administração de 1,2 a 2,0CAM de sevofluorano. Para Nakaigawa et al. (1995), esse fármaco não promove alterações no sistema de condução cardíaca e é importante na estabilidade do ritmo cardíaco durante a anestesia.

Segundo Bernard et al., 1990, o sevoflurano tende a preservar o débito cardíaco em concentrações inferiores a 1,2CAM. Mutoh et al. (1995) relataram a ocorrência do aumento desse parâmetro durante a indução da anestesia pela inalação rápida do fármaco. De maneira semelhante ao que ocorre com os demais anestésicos voláteis, o sevofluorano, em concentrações elevadas, promove diminuição progressiva da pressão arterial sangüínea e, mesmo em concentrações baixas, é capaz de potencializar quadros hipotensivos (Lowe et al., 1996). 
Segundo Shepherd (1996) a adrenalina apresenta ação estimulante sobre receptores adrenérgicos $\alpha$ e $\beta$ levando a vasoconstricção. Nunes (1995) relata que a atividade dessa catecolamina nos nódulos sinoatrial e atrioventricular, bem como no miocárdio, é responsável pela ocorrência de bloqueios.

O objetivo deste estudo foi avaliar os efeitos da dexmedetomidina sobre o ritmo cardíaco e a pressão arterial em cães anestesiados com sevofluorano e submetidos a doses crescentes de adrenalina.

\section{MATERIAL E MÉTODOS}

Foram utilizados 20 cães, de ambos os sexos, sem raça definida, adultos considerados sadios, distribuídos aleatoriamente em dois grupos de 10 animais cada um: grupo placebo (GP) e grupo dexmedetomidina (GD). Os animais foram cedidos pelo canil da FCAV-UNESP e não foram utilizadas fêmeas prenhes ou em estro. Antes dos procedimentos, todos os animais foram mantidos em jejum alimentar de 12 horas e hídrico de três horas.

O GP recebeu, por via intravenosa (IV), solução de $\mathrm{NaCl}$ a $0,9 \%$, na dose de $0,3 \mathrm{ml} / \mathrm{kg}$. Após 10 minutos, os animais foram induzidos à anestesia, com a administração de sevofluorano ${ }^{1}$ por meio de máscara facial vedada, até a perda do reflexo laringotraqueal. Em seguida, os animais foram intubados com sonda de Magill, de diâmetro adequado ao porte do animal, imediatamente acoplada ao aparelho de anestesia inalatória para administração do sevofluorano em circuito valvular com reinalação parcial de gases ${ }^{2}$.

A manutenção da anestesia foi realizada com sevofluorano, diluído em oxigênio, em fluxo total de $30 \mathrm{ml} / \mathrm{kg} / \mathrm{min}$, na concentração alveolar mínima (CAM) de 1,5, mensurada no ar expirado, por meio de analisador de gases $^{3}$, considerando-se a CAM, em cães, equivalente a $2,3 \mathrm{~V} \%$.

\footnotetext{
${ }^{1}$ Sevorane - Cristália Produtos Químicos e Farmacêuticos Ltda.

${ }^{2}$ Datex Ohmeda - mod Excel 210 SE - Processo FAPESP 97/10668-4

${ }^{3}$ Datex Ohmeda - mod. RGM 5250 - Processo FAPESP 96/12830-0
}

Decorridos vinte minutos do início da indução da anestesia, foi iniciada a administração IV, por meio de bomba de infusão ${ }^{4}$, de adrenalina $^{5}$, $0,02 \mathrm{mg} / \mathrm{ml}$ de $\mathrm{NaCl}$ a $0,9 \%$, na dose inicial de $1 \mu \mathrm{g} / \mathrm{kg} /$ minuto. A partir de 10 minutos do início da administração da catecolamina, a dose foi acrescida em uma unidade, cessando em $5 \mu \mathrm{g} / \mathrm{kg} / \mathrm{minuto}$.

Para o GD, empregou-se a mesma metodologia, substituindo-se a solução de $\mathrm{NaCl}$ a $0,9 \%$ por hidrocloridrato de dexmedetomidina ${ }^{6}$, na dose de $1 \mu \mathrm{g} / \mathrm{kg}$.

Os períodos de observação tiveram início imediatamente antes (M0) e após a aplicação do fármaco ou solução de $\mathrm{NaCl}$ 0,9\% (M1). Novas mensurações ocorreram 10 minutos a partir do inicio da infusão contínua de adrenalina (M2) repetindo-se as mensurações em intervalos de 10 minutos, durante 40 minutos (M3 a M6).

Foram avaliadas a freqüência cardíaca (FC), nos momentos M0 a M6, em eletrocardiógrafo computadorizado ${ }^{7}$ ajustado para mensurações na derivação DII e calculadas a partir do registro do intervalo R-R; arritmias obtidas por observação da onda eletrocardiográfica na derivação DII a partir de M2 até M6, observando-se a ocorrência de bloqueios atrioventriculares (BAV) e complexos ventriculares prematuros (ESV). As pressões arteriais, sistólica (PAS), diastólica (PAD) e média (PAM) foram mensuradas pelo método oscilométrico, em $\mathrm{mmHg}$, nos tempos M0, M2, M3, M4, M5 e M6) utilizando-se monitor multiparamétrico ${ }^{8}$, cujo manguito, de tamanho proporcional ao diâmetro dos membros dos animais, foi adaptado ao membro torácico, acima da articulação do cotovelo e sobre a artéria braquial. As aferições foram feitas em triplicata e o valor final obtido pela média aritmética.

Para a avaliação estatística adotou-se a análise de variância (ANOVA), seguida pelo teste de Tukey $(\mathrm{P}<0,05)$.

\footnotetext{
${ }^{4}$ Lifemed - mod. Fars 600 - Processo FAPESP 95/2763-1

${ }^{5}$ Cloridrato de adrenalina $1 / 1000$ - Laboratório Santista Ltda.

${ }^{6}$ Precedex - Abbot Laboratórios do Brasil Ltda.

${ }^{7}$ Teb - mod. ECGPC software versão 1.10 - Processo FAPESP 96/1151-5

${ }^{8}$ Dixtal Biomédica Ind. Com. Ltda - DX - 2010-LCD -

Processo FAPESP 02/
} 
Para as variáveis BAV e ESV utilizou-se o delineamento em parcelas subdivididas com dois grupos (GD e GP) e cinco momentos de avaliação (M2 a M6). Os valores observados foram transformados em $\log (\mathrm{X}+1)$ com o objetivo de normalizar os dados e homogeneizar as variâncias.

\section{RESULTADOS E DISCUSSÃO}

Entre os grupos, a FC no GD apresentou redução significativa dos valores observados no momento inicial (M1) imediatamente após a administração do fármaco (Tab. 1). Dentro dos grupos, no GD pôde-se verificar que houve redução dos valores iniciais, a partir de M1 até trinta minutos após o início da infusão de adrenalina (M4), quando houve início de acréscimo dos valores sem diferenciar-se dos basais até o final da avaliação (M6). Com essa redução, os valores ficaram abaixo da faixa de normalidade para a espécie. Os resultados obtidos neste estudo corroboram os achados de Sabbe et al (1994) ao descreverem que a administração IV de dexmedetomidina resulta em redução no ritmo cardíaco.

Tabela 1. Freqüência cardíaca (FC), pressões arteriais: sistólica (PAS), média (PAM) e diastólica (PAD) (média \pm desvio-padrão) de cães pré-tratados com dexmedetomidina (GD) ou solução de $\mathrm{NaCl}$ a $0,9 \%$ (GP) e anestesiados pelo sevofluorano, submetidos à arritmia cardíaca induzida pela adrenalina

\begin{tabular}{|c|c|c|c|c|c|c|c|c|}
\hline & & M0 & M1 & M2 & M3 & $\mathrm{M} 4$ & M5 & M6 \\
\hline \multirow{2}{*}{$\underset{\text { (bat/min) }}{\mathrm{FC}}$} & GD & $117,0 \pm 26,3 a$ & $66,0 \pm 14,8 \mathrm{Ab}$ & $67,0 \pm 18,8 \mathrm{~b}$ & $58,0 \pm 9,6 \mathrm{~b}$ & $69,0 \pm 17,2 \mathrm{ab}$ & $81,0 \pm 21,3 \mathrm{a}$ & $90,0 \pm 23,2 \mathrm{a}$ \\
\hline & GP & $107,0 \pm 23,4 \mathrm{a}$ & $108,0 \pm 16,7 \mathrm{Ba}$ & $78,0 \pm 16,7 \mathrm{a}$ & $65,0 \pm 11,5 \mathrm{~b}$ & $66,0 \pm 10,6 \mathrm{~b}$ & $77,0 \pm 18,3 \mathrm{ab}$ & $86,0 \pm 21,0 \mathrm{ab}$ \\
\hline \multirow{2}{*}{$\begin{array}{c}\mathrm{PAS} \\
(\mathrm{mmHg})\end{array}$} & GD & $124,3 \pm 23,8 \mathrm{a}$ & & $97,2 \pm 21,2 \mathrm{a}$ & $126,8 \pm 30,5 \mathrm{a}$ & $163,6 \pm 21,3 \mathrm{Ab}$ & $177,8 \pm 30,9 \mathrm{bc}$ & $197,3 \pm 36,8 \mathrm{c}$ \\
\hline & GP & $131,6 \pm 23,1 \mathrm{ab}$ & & $107,9 \pm 19,7 \mathrm{a}$ & $118,7 \pm 18,8 \mathrm{ab}$ & $127,3 \pm 28,2 \mathrm{Bab}$ & $151,7 \pm 39,6 \mathrm{ab}$ & $177,8 \pm 35,4 \mathrm{c}$ \\
\hline \multirow{2}{*}{$\begin{array}{c}\text { PAM } \\
(\mathrm{mmHg})\end{array}$} & GD & $89,5 \pm 23,9 \mathrm{a}$ & & $71,8 \pm 15,6 \mathrm{a}$ & $99,0 \pm 25,9 \mathrm{a}$ & $137,3 \pm 23,5 \mathrm{Ab}$ & $153,8 \pm 34,7 \mathrm{bc}$ & $176,0 \pm 47,7 \mathrm{c}$ \\
\hline & GP & $97,7 \pm 17,0 \mathrm{ab}$ & & $80,2 \pm 20,1 \mathrm{a}$ & $87,0 \pm 19,4 a b$ & $95,1 \pm 28,0 \mathrm{Bab}$ & $121,2 \pm 34,6 a b$ & $133,3 \pm 26,1 \mathrm{c}$ \\
\hline \multirow{2}{*}{$\begin{array}{c}\mathrm{PAD} \\
(\mathrm{mmHg})\end{array}$} & GD & $72,3 \pm 19,4 a$ & & $56,6 \pm 16,6 \mathrm{a}$ & $79,2 \pm 27,1 \mathrm{a}$ & $118,3 \pm 25,3 \mathrm{Ab}$ & $137,0 \pm 37,9 \mathrm{bc}$ & $161,6 \pm 56,1 \mathrm{c}$ \\
\hline & GP & $77,3 \pm 16,1 \mathrm{ab}$ & & $58,1 \pm 17,0 \mathrm{a}$ & $68,5 \pm 22,4 \mathrm{ab}$ & $75,6 \pm 28,0 \mathrm{Bab}$ & $99,9 \pm 33,2 \mathrm{ab}$ & $109,5 \pm 30,1 \mathrm{c}$ \\
\hline
\end{tabular}

Médias seguidas por letras distintas, minúsculas na linha e maiúsculas na coluna, diferem entre si pelo teste de Tukey (P<0,05). MO e M1: antes e após tratamento com dexmedetomidina ou $\mathrm{NaCl}$ a 9\%; M2 a M6: mensurações a intervalos de 10minutos.

No GP, foi observada redução dos valores iniciais da $\mathrm{FC}$ vinte minutos após o início da infusão de adrenalina (M3). Possivelmente, em decorrência da estabilidade proporcionada pelo sevofluorano, a FC não se elevou consideravelmente em nenhum momento, em ambos os grupos. Relatos, sobre a estabilidade conferida por esse fármaco, foram feitos por Ebert (1996) e Hettrick et al. (1996).

Em relação aos BAV, apesar de não terem sido significativos, os valores no GD foram maiores que no GP em M2 e M3 (Tab. 2). Este resultado sugere a ação dos agonistas de receptores alfa-2 em alterarem a atividade simpática e a parassimpática nas células de condução atrioventricular e promover despolarização espontânea dos tecidos do átrio (Tranquilli, 2004). Em que pese a provável influência da dexmedetomidina no desenvolvimento dos BAV, vale ressaltar o potencial efeito da adrenalina, na alteração da condução atrioventricular conforme citado por Nunes (1995). De acordo com Lemke et al. (1993), a ocorrência de bloqueios atrioventriculares está relacionada ao mecanismo de escape potencializado pelo reflexo vagal, pois, o tono vagal medeia o estímulo de barorreceptores e diminui a taxa de despolarização do nodo sinoatrial, o que resulta em bloqueios atrioventriculares.

$\mathrm{Na}$ análise individual, houve redução do número de BAV a partir de M4 até o final da avaliação nos dois grupos (Tab. 2), fato este ocorrido em virtude da estabilidade do ritmo cardíaco proporcionada pelo sevoflurano, o qual não interfere no sistema de condução cardíaca (Nakaigawa et al., 1995).

Com relação ao número de ESV, ressalta-se a considerável variabilidade entre os animais nos grupos, achado que, segundo Nunes et al. (1999), está relacionado à diferente sensibilidade individual do miocárdio a determinados fármacos, em especial à adrenalina.

$\mathrm{Na}$ análise dos grupos, observou-se aumento dos valores médios de ESV no GP em M3 e M4, em relação ao GD (Tab. 2). Este fato sugere que a dexmedetomidina reduz a ocorrência de complexos ventriculares precoces originários de focos ectópicos indicando uma provável ação 
protetora do fármaco sobre o miocárdio. Estes resultados foram similares aos obtidos por Iida et al. (2006), ao analisarem os efeitos da dexmedetomidina IV associada à fenilefrina durante a ressuscitação cardiopulmonar em cães, concluíram que o $\alpha-2$ agonista reduz o total de choques necessários à desfibrilação bem como o número batimentos ventriculares ectópicos após a ressuscitação, auxiliando na estabilidade da circulação sistêmica.

Tabela 2. Bloqueio atrioventriculare (BAV) e complexo ventricular prematuro (ESV) em cães prétratados com dexmedetomidina (GD) ou solução de $\mathrm{NaCl}$ a $0,9 \%$ (GP) e anestesiados pelo sevofluorano, submetidos à arritmia cardíaca induzida pela adrenalina

\begin{tabular}{|c|c|c|c|c|c|c|}
\hline & & M2 & M3 & M4 & M5 & M6 \\
\hline & GD & $70,5 a$ & $53,0 \mathrm{ab}$ & $11,6 \mathrm{bc}$ & $2,9 \mathrm{c}$ & \\
\hline $\begin{array}{c}\text { BAVs } \\
(\log \\
\mathrm{X}+1)\end{array}$ & GP & $42,2 \mathrm{a}$ & $29,3 \mathrm{ab}$ & $16,9 b c$ & $6,1 \mathrm{c}$ & \\
\hline & GD & $151,8 \mathrm{a}$ & $170,4 \mathrm{Aa}$ & $119,1 \mathrm{Aa}$ & $92,3 a$ & $15,1 \mathrm{~b}$ \\
\hline $\begin{array}{c}\text { ESVs } \\
(\log \\
\mathrm{X}+1)\end{array}$ & GP & $112,1 \mathrm{a}$ & $213,0 \mathrm{Ba}$ & $220,3 \mathrm{Ba}$ & $78,4 a$ & $1,4 b$ \\
\hline
\end{tabular}

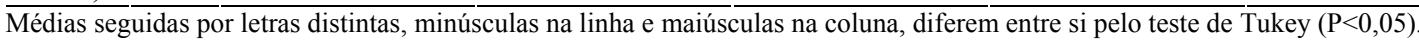
M2 a M6: mensurações a intervalos de 10minutos.

BAV: coeficiente de variação $(\mathrm{CV})$ grupo $(\mathrm{G})=140,85 \%$; $\mathrm{CV}$ momento $(\mathrm{M})=112,05 \%$; $\mathrm{dms}(\mathrm{G})=0,78 \% ; \mathrm{dms}(\mathrm{M})=1,31 \%$. ESV: $\mathrm{CV}(\mathrm{G})=101,78 \% ; \mathrm{CV}(\mathrm{M})=52,43 \% ; \operatorname{dms}(\mathrm{G})=1,31 \% ; \operatorname{dms}(\mathrm{M})=1,42 \%$.

Individualmente no GD houve redução dos valores iniciais de ESV observados em M6 e no GP, após discreto aumento inicial das médias em M3, houve redução significativa em M6. A redução dos valores de ESV, ao final do período experimental nos dois grupos, sugere que houve saturação de receptores, provavelmente do tipo $\alpha$ (Rezende et al., 2000) associado ao efeito antiarritmogênico do sevofluorano (Novalija et al., 1998), pois houve redução da quantidade de complexos ventriculares ectópicos, a partir da dose de $3 \mu \mathrm{g} / \mathrm{kg} / \mathrm{min}$ de adrenalina.

A PAS, PAM e PAD apresentaram diferença somente em M4, onde a média do GD foi superior à do GP, o que provavelmente está relacionado à diminuição da concentração plasmática da dexmedetomidina (Sabbe et al., 1994), reduzindo, dessa forma, sua atividade hipotensora, conforme observação de Fantoni e Cortopassi (2002), ao relatarem a ação dos agonistas de receptores alfa- 2 adrenérgicos sobre a pressão arterial. Na análise individual dos grupos, os valores de PAS, PAM e PAD do GD apresentaram aumento em M4, persistindo até o término do período experimental em M6. No GP foi observado aumento dos valores iniciais em M6 (Tab. 1). O aumento, nos momentos finais da avaliação experimental, pode ter ocorrido devido à ação vasoconstritora predominante da adrenalina (Shepherd 1966).

\section{CONCLUSÕES}

A dexmedetomidina interfere de maneira importante na condução atrioventricular levando à maior ocorrência de bloqueio atrioventricular e reduz o número de complexo ventricular prematuro nas doses infundidas de 2 e $3 \mu \mathrm{g} / \mathrm{kg} / \mathrm{min}$ de adrenalina. Complementarmente o $\alpha_{2}$ agonista produz redução da pressão arterial e da freqüência cardíaca.

\section{REFERÊNCIAS BIBLIOGRÁFICAS}

BERNARD, J.M.; WOUTHERS, P.F.; DOURSOUT, M.F. et al. Effects of sevoflurane and isoflurane on cardiac and coronary dynamics in chronically instrumented dogs. Anesthesiology, v.72, p.659-662, 1990.

EBERT, T.J. Cardiovascular and autonomic effects of sevoflurane. Acta Anaesthesiol. Belg. v.47, p.15-21, 1996. 
FANTONI, D.T.; CORTOPASSI, S.R.G. Medicação pré-anestésica. In: FANTONI, D.T.; CORTOPASSI, S.R.G. (Eds). Anestesia em cães e gatos. São Paulo: Roca, 2002. p.151-158.

GABAS, D.T.; OLIVA, V.N.L.S.; MATSUBA, S.H.V. et al. Estudo clínico e cardiorrespiratório em cadelas gestantes com parto normal ou submetidas à cesariana sob anestesia inalatória com sevofluorano. Arq. Bras. Med. Vet. Zootec., v.58, p.518-524, 2006.

HETTRICK, D.A.; PAGEL, P.S.; WARLTIER, D.C. Desflurane, sevoflurane and isoflurane impair canine left ventricular-arterial coupling and mechanical efficiency. Anesthesiology, v.85, p.404-413, 1996.

IIDA H., IIDA M. OHATA, H. et al. Effects of dexmedetomidine on cerebral circulation and systemic hemodynamics after cardiopulmonary resuscitation in dogs. J. Anesth., v.20, p.202-207, 2006.

KUUSELA, E.; RAEKALLIO, M.; VAISANEN, M. et al. O. Comparison of dexmedetomidine as premedicants in dogs undergoing propofolisoflurane anesthesia. Am. J. Vet. Res., v.62, p.1073-1079, 2001.

LEMKE, K.A.; TRANQUILLI, W.J.; THURMON, J.C. et al. Alterations in the arrythmogenic dose of epinephrine after xylazine or medetomidine administration in isofluoraneanesthetized dogs. Am. J. Vet. Res., v.54, p.21392144, 1993.

LOWE, D.; HETTRICK, D.A.; PAGEL, P.S. et al. Influence of volatile anesthetics on left ventricular afterload in vivo. Differences between desflurane and sevoflurane. Anesthesiology, v.85, p.112-120, 1996.

MUTOH, T.; NISHIMURA, R.; KIM, H. et al. Rapid inhalation induction of anesthesia by halotano, enflurane, isoflurane and sevoflurane and their cardiopulmonary effects in dogs. J. Vet. Med. Sci., v.57, p.1007-1013, 1995.

MUTOH, T.; NISHIMURA, R.; KIM, H et al. Cardiopulmonary effects of sevoflurane, compared with halothane, enflurane and isoflurane, in dogs. Am. J. Vet. Res., v.58, p.885890, 1997.

NAKAIGAWA, Y.; AKAZAWA, S.; SHIMIZU, $\mathrm{R}$. et al. Comparison of the effects of halotane, isoflurane, and sevoflurane on atrioventricular conduction times in pentobarbital-anesthetized dogs. Anesth. Analg., v.81, p.249-53, 1995.

NOVALIJA, E., HOGAN, Q.H., KULIER, A.H. et al. Effects of desflurane, sevoflurane and halothane on postinfarction spontaneous dysrhythmias in dogs. Acta Anaesth. Scand., v.42, p.353-357, 1998.

NUNES, N. Efeitos cardiocirculatórios da associação de levomepromazina e quetamina, em cães (Canis familiaris) pré-tratados ou não pelo propranolol. 1995. 142f. Tese (Doutorado) Faculdade de Medicina Veterinária e Zootecnia, Universidade Estadual Paulista, Botucatu.

NUNES, N.; SANTOS, P.S.P.; REZENDE, M.L. et al. Emprego da levomepromazina no bloqueio da arritmia cardíaca induzida pela adrenalina, em cães anestesiados pelo halotano. ARS Vet., v.15, p.164-169, 1999.

REZENDE, M.L.; SANTOS, P.S.P.; NUNES, N. et al. Emprego da levomepromazina no bloqueio da arritmia induzida pela adrenalina, em cães anestesiados pelo sevoflurano. Cienc. Rural, v.30, p.421-424, 2000.

SABBE, M.B.; PENNING, J.P.; OZAKI, G.T. et al. L. Spinal and systemic action of the alpha 2 receptor agonist dexmedetomidine in dogs. Antinociception and carbon dioxide response. Anesthesiology, v.80, p.1057-1072, 1994.

SCHEININ, H.; VIRTANEN, R. Medetomidine, a novel alfa 2 adrenoceptor agonist: a review of pharmacodinamic effects. Prog. Neuropsychopharmacol. Biol. Psychiatry, v.13, p.635-651, 1989.

SHEPHERD, J.T. Role of the veins in the circulation. Circulation, v.32, p.484, 1966.

TRANQUILLI, W.J. $\alpha-2$ Agonistas. In: GREENE, S.A. (Ed). Segredos em anestesia veterinária e manejo da dor. 1.ed. São Paulo: Artmed, 2004. p.107, 2004. 